\title{
Reviews
}

\section{Single-cell Analysis with Microfluidic Devices}

\author{
Xiaowen Ou, Peng Chen, and Bi-Feng LIU ${ }^{\dagger}$ \\ The Key Laboratory for Biomedical Photonics of MOE at Wuhan National Laboratory for Optoelectronics- \\ Hubei Bioinformatics \& Molecular Imaging Key Laboratory, Systems Biology Theme, Department of Biomedical \\ Engineering, College of Life Science and Technology, Huazhong University of Science and Technology, \\ Wuhan 430074, China
}

\begin{abstract}
A microfluidic device as a pivotal research tool in chemistry and life science is now widely recognized. Indeed, microfluidic techniques have made significant advancements in fundamental research, such as the inherent heterogeneity of single-cells studies in cell populations, which would be helpful in understanding cellular molecular mechanisms and clinical diagnosis of major diseases. Single-cell analyses on microdevices have shown great potential for precise fluid control, cell manipulation, and signal output with rapid and high throughput. Moreover, miniaturized devices also have open functions such as integrating with traditional detection methods, for example, optical, electrochemical or mass spectrometry for single-cell analysis. In this review, we summarized recent advances of single-cell analysis based on various microfluidic approaches from different dimensions, such as in vitro, ex vivo, and in vivo analysis of single cells.
\end{abstract}

Keywords Microfluidics, single-cell analysis, in vitro, ex vivo, in vivo

(Received January 10, 2019; Accepted February 25, 2019; Advance Publication Released Online by J-STAGE March 8, 2019)

1 Introduction

2 Single-cell in vitro

$2 \cdot 1$ Analysis of DNA and genome

2.2 Analysis of RNA and transcriptome

2.3 Analysis of protein and proteome

2.4 Analysis of metabolite and metabolome

3 Single-cell ex vivo
609

610

$3 \cdot 2$ Electrochemical analysis

4 Single-cell in vivo

615

5 Conclusions

6 Acknowledgements

7 References
615

616

616

\section{Introduction}

As completion of the Human Genome Project in the past two decades, rapid development on genomics, transcriptomics, proteomics and metabolomics were realized, and have made great progress in chemistry and life science. ${ }^{1}$ However, most of advancements were acquired at cell populations or tissues level without consideration of cell heterogeneity. Due to the heterogeneity in cellular events, such investigations have

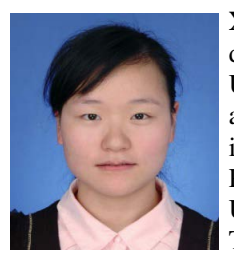

Xiaowen OU received her BS degree from Hubei Normal University, China in 2012, and received her Ph.D. degree in Polymeric Chemistry and Physics from Huazhong University of Science and Technology (HUST, China) in 2017. She then working with Prof. Bi-Feng Liu, as a postdoctoral research associate at HUST. Her current research interest are focused on the microfluidics and biosensors for early detection of cancer biomarkers

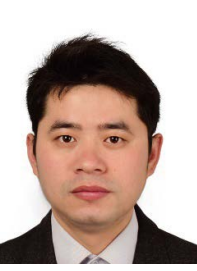
Currently, he is a Postdoctoral Research Associate at the HUST working with Prof. Bi-Feng Liu. His research interest include microfluidic-based methods for cell analysis, exosomes related early cancer diagnosis \& therapy and point-of-care testing (POCT).

Peng CHen received his BS degree from University of Jinan, China, in July 2010 and then received his Ph.D. degree in Biomedical Engineering from Huazhong University of Science and Technology in Prof. Terabe's group (Japan) as a JSPS postdoctoral fellow. He joined HUST as a professor in 2003. He is now serving as Executive Dean of the College of Life Science \& Technology, HUST. His research focuses on systems biologyoriented analytical science in the areas of microfluidics, mass spectrometry coupled microseparation and molecular imaging.

$\dagger$ To whom correspondence should be addressed.

E-mail: bfliu@mail.hust.edu.cn 
A

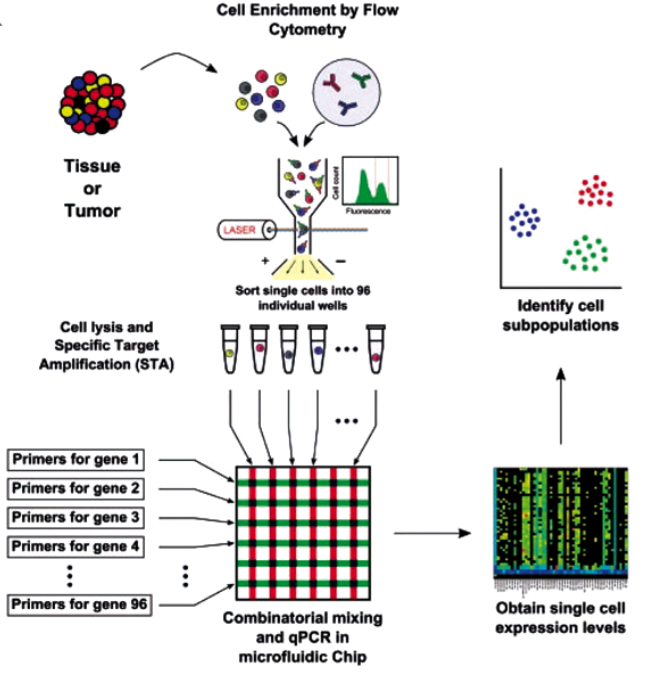

B a
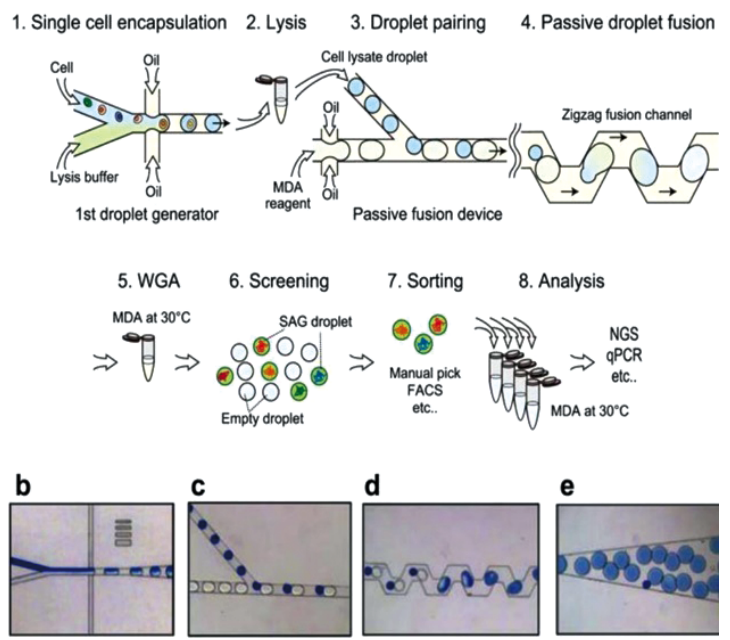

Fig. 1 Microfluidic device for single-cells genome profiling. (A) Sketch of a typical single-cell qPCR workflow performed with the Fluidigm Dynamic Array microfluidic chip. Adapted with permission from Ref. 23, Copyright 2017 Oxford University Press. (B) (a) Workflow of sd-MDA and subsequent single cell genome analysis, (b,c,d and e) Microphotographs of (b) the 1st droplet generator, (c) droplet pairing channel, (d) following zigzag channel, and (e) downstream channel in the passive fusion device. Adapted with permission from Ref. 24, Copyright 2017 Springer Nature Publishing AG.

recently been scaled down to the single-cell level. Analyzing the heterogeneities of individual cells in the morphology, composition and functions is important for detecting cell-based diseases and determining differences from many biological systems, and the analyses of single cells at muti-dimensions can also reveal an effect of different life conditions, cell-cycle, and the surrounding environment. ${ }^{2}$ Thus, various types of traditional methods including flow cytometry, microcolumn liquid chromatography, capillary electrophoresis, spectroscopy and electrochemistry for single-cell analysis have been developed, ${ }^{3-7}$ showing respectable advantages, such as high sensitivity and accuracy. However, most of them always encounter the difficulty of isolating, sorting and operating on a single-cell level in the process of sample preparation. To address these issues, microfluidic chips where their dimensions are on the same scale as those of cells were emerged, which have proven to be fascinating devices for single-cell analysis with precise fluid control, cell manipulation, and signal output. ${ }^{8-13}$ Due to the flexible design of the chip architecture according to different demands, single-cell isolating and sorting can be conducted by various modes such as mechanical, hydrodynamic, dielectrophoresis, droplet methods and so on. In addition, the microfluidic devices have the merits of automation, parallel processing, large surface-to-volume ratio and portability. This allows the integration of multiple liquid handling processes, such as pumping, sampling, dispensing and washing, resulting in the requirement of little reagent consumption and substantially reduces the labor work relative to the conventional experimental installation. Moreover, miniaturized devices have also open functions, such as integrating with traditional detection methods, for example, optical, electrochemical or mass spectrometry (MS) for single-cell analysis with high sensitivity and high throughput. ${ }^{14-19}$ Recently, many reviews have elaborated the single-cell isolation and lysis for microfluidic analysis. ${ }^{20,21}$ In this review, we focus on current research from different dimensions (in vitro, ex vivo and in vivo) for single-cell analysis on microfluidic devices.

\section{Single-cell in vitro}

In the past years, the capability of "omics" technology has been well studied and further interest has evolved for single-cell analysis at the molecular level, which could provide exciting new insights into genomics, transcriptomics, proteomics, and metabonomics. The emerging microfluidic technology with the capacity to manipulate a small volume of sample in microchannels, enables single-cell analysis events to be manipulated and dynamically characterized in a confined microenvironment. These features of microfluidic devices make them well suited for the analysis of single-cell together with other signal amplification systems, such as, polymerase chain reaction (PCR). Here, we highlight several examples in the genome, transcriptome, proteome, metabonome analysis of single cells by microfluidic devices.

\subsection{Analysis of DNA and genome}

Single-cell genomics permit investigations of cellular diversity in a wide field of biological species. Until recently, the method of quantitative real-time PCR (qPCR) is the most advanced technique to study gene expression and sequence information of single cells, since its highly sensitive for only one gene molecule detection. ${ }^{22}$ In addition, due to the wide dynamic range and good reproducibility of qPCR, its quite suitable for quantifying selected gene species from single cells. However, the simultaneous detection of multiple genes from many different individual cells requires vast parallel biochemical reactions. Microfluidic devices with the merits of high parallelization and combinatorial mixing solve this problem well. The progress of microfluidic-based qPCR is shown in Fig. 1A. ${ }^{23}$ Firstly, individual cells were sorted into 96 well plates filled with PCR buffer by a flow cytometer, and dissociated by the hypotonic pressure of cell membranes from influx of fluids. Then, specific target transcript amplifications were performed by PCR use a mixture of primers for a predetermined set of genes in microfluidic chip. After that, the expression levels of selected 

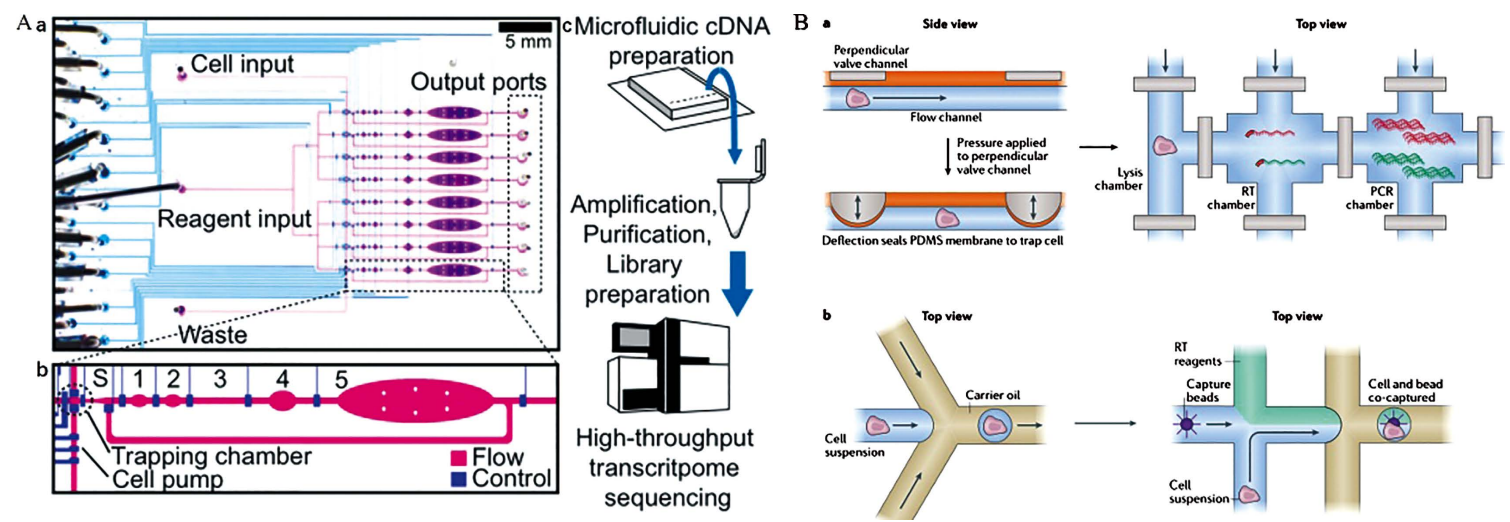

Fig. 2 Microfluidic device for single-cells transcriptome profiling. (A) (a) Micrograph of the microfluidic device filled with colored dye. (b) Detailed diagram of a single-reaction pipeline. (c) Complete experimental pipeline. Adapted with permission from Ref. 34, Copyright 2014 National Academy of Sciences. (B) Overview of the major microfluidic device types: valves and droplets. Adapted with permission from Ref. 37, Copyright 2017 Springer Nature Publishing AG.

genes could be measured use benchtop qPCR in tubes or Fluidigm Dynamic Arrays. Using this assay, a mass of independent qPCR reactions could be completed in one experiment with the parallelization of microfluidic.

In the past decade, microfluidic droplet systems due to the automation of droplet manipulation and emulsification with a chemically closed reaction environment have become a favorable technique for addressing single cells and intracellular component analysis together with qPCR. For example, Hosokawa et al. ${ }^{24}$ integrated the $\mathrm{qPCR}$ with a droplet microfludic device realized whole genome amplification (WGA) of single cells (Fig. 1B). In this strategy, a good deal of single cells were encapsulated into the droplet generator and separated in the droplets with cell lysis buffer. After the addition of multiple displacement amplification (MDA) reagent to each droplet, passive droplet fusion was produced and the fusional droplets were re-injected into the microchannels and incubated for a single cells WGA through the qPCR technique. Besides qPCR, the amplification system of high-throughput on-chip and digital PCR (dPCR) are also significant for single-cell genomic analysis due to being sensitive for the quantification of a target DNA molecule and the identification of rare variants by taking advantage of less dilution of the starting sample. For instance, Kiss et al. ${ }^{25}$ introduced on-chip dPCR, where fluorescent DNAs distinguish the original product, and provide a digital readout of the proportion of a mutant sequence in the total, to detect singlecopy target DNA in picoliter droplet reservoirs using a continuous flow-based strategy. Heyries et al. ${ }^{26}$ demonstrated an integrated microfluidic device for the high-throughput dPCR analysis of single cells in $\mu \mathrm{L}$ reactions. Several other reviews for amplification assays of enzyme-based are recommended for further understanding. ${ }^{27,28}$

\subsection{Analysis of RNA and transcriptome}

The transcriptome is an essential section of cell identity, since RNA plays a critical role as a messenger. ${ }^{29,30}$ Hence, understanding the transcriptome of individual cells is significant to characterize complex biological systems. A next-generation sequencing technique is a favorable approach for transcriptome analysis. $^{31}$ However, under certain conditions, only a small quantity of sample is available, which needs highly sensitive methods that can preferably be used at the single-cell level. Until 2009, Tang et al. ${ }^{32}$ first proposed the conception of single- cell RNA-sequencing (scRNA-seq) whole-transcriptome analysis, using only a single mouse blastomere could detect expression of 5270 more genes than microarrays using hundreds of blastomeres. Inspired by Tang2009 protocol $^{33}$ for reverse transcription and cDNA amplification, Huang et al. ${ }^{34}$ presented a microfluidic-based system for single-cell whole-transcriptome sequencing. As shown in Fig. 2A, single cells were captured, sorted, and lysed in multilayer microfluidic devices with three integrated valves, which positioned over corresponding reaction chambers. These pressure valves regulated the reagent to optimize mixing in the swelled chamber, where mRNAs with poly(A) tails were reverse-transcribed into cDNA. cDNA libraries representing whole single-cell transcriptomes were then collected and sequenced using a next-generation sequencing platform. For a further introduction to readers, Xie et al. ${ }^{35}$ summarized a review elaborating the methodology and applications of single-cell whole-genome amplification and sequencing.

scRNA-seq is a critical step in single-cell gene expression, and can be used to dissect transcriptomic heterogeneity by investigating expression profiles at the cell level. The current techniques face practical challenges when scaling to tens of thousands of cells, or when it is necessary to capture as many cells as possible from a limited sample. The first published platform allow people to handle a number of cells was termed CytoSeq. Fan et al. ${ }^{36}$ used this technique to segregate a human PBMC population of about 750 cells into major cells types by combining next-generation sequencing with stochastic barcoding of single cells for gene expression. Only months after the CytoSeq paper appeared, two further single-cell transcriptome analysis studies based on droplet technology were published continuously by Weitz and co-workers. They named this technique as Drop-seq, a strategy for quickly profiling thousands of individual cells by separating them into nanoliter-sized aqueous droplets, associating a different barcode with each cell's RNAs, and sequencing them all together. As shown in Fig. 2B, ${ }^{37}$ individual cells and microparticles (beads) that deliver barcoded primers were encapsulated in droplets. From Dropseq system, they analyzed transcriptomes from 44808 mouse retinal cells and identified 39 transcriptionally distinct cell populations, creating a molecular atlas of gene expression for known retinal cell classes and novel candidate cell subtypes. ${ }^{38}$ 

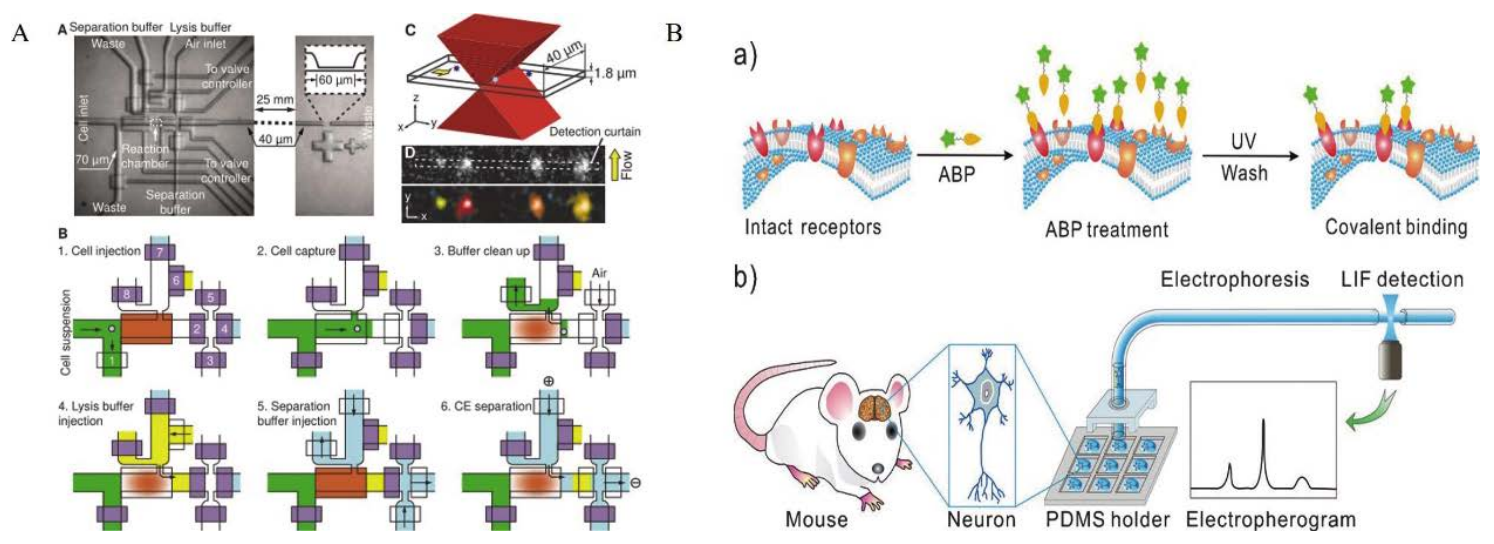

Fig. 3 Microfluidic device for single-cells proteome profiling. (A) Graphical representation of the single-cell analysis chip for counting low-copy number proteins. Adapted with permission from Ref. 44, Copyright 2007 American Association for the Advancement of Science. (B) Illustration of the SCCP strategy. a) Activity-based probe (ABP) labeling procedure. b) Capillary electrophoresis-laser induced fluorescence (CE-LIF) detection of the labeled single cells. Adapted with permission from Ref. 46, Copyright 2014 Wiley-VCH Verlag GmbH \& Co. KGaA, Weinheim.

\subsection{Analysis of protein and proteome}

In biological processes, proteins are important for regulating cellular function, controlling cell growth and transporting molecules across cell membranes. Therefore, confirming the quantity and activity of proteins is important for understanding the cellular molecular mechanisms. Studying DNA or RNA expression can offer qualitative information on the gene products-proteins, but can not offer information about the quantification of protein. Proteomic measurements are very complicated, since there are various types of measurements to be made, such as enzymatic activity, protein-protein interactions, protein abundance, protein secretion, protein translocation and post-translational modifications. ${ }^{39}$ So, methods and tools to directly research the modifications and interactions of proteins in a single cell are necessary. Since 2004, Dovichi and Nolan first proposed the concept of single-cell proteomics (SCP), ${ }^{40,41}$ a wide varity of techniques for SCP analysis were developed, the most common of which are chemical cytometry and flow cytometry. ${ }^{42,43}$ Flow cytometry based on multiple parameters shows great potential in mapping cellular signal transduction networks and investigating the molecular mechanisms of diseases, but ignoring the heterogeneity among single cells. The heterogeneity among single cells can be readily discriminated by chemical cytometry. After that, Zare et al..$^{44}$ proposed a method to count low-copy number proteins in a single cell by a microfluidic platform. As shown in Fig. 3A, on this integrated device, a single cell could be lysed, labled and separated, after that, the protein contents was quantified through single-molecule fluorescent-antibody binding. Herr et al. ${ }^{45}$ constructed a brand new microfluidic SCP approach for high-throughput single cell Western blotting, which overcame the limitation of antibody fidelity and constituted a versatile tool for the study of complex cell populations at single-cell resolution.

Recently, our group designed a smart single-cell chemical proteomics (SCCP) platform to identify low-copy membrane proteins on single primary neurons. ${ }^{46}$ As shown in Fig. 3B, through the specifically designed activity-based probe (ABP), the transmembrane receptors (GB1 as the target membrane protein in this assay) were targeted on single primary neurons extracted from a mouse. The single cells functionally labeled with $\mathrm{ABP}$ were encapsulated in droplets that were stored in a PDMS microwell holder for further capillary electrophoresis and laser-induced fluorescence detection of the targeted proteins. The results showed that the GB1 splicing variants in MEF and HEK cells were expressive, and the previously study only revealed at the transcriptional level. We also used this strategy to identify functional proteins in the lysosome of tumors in another work..$^{47}$ More recently, Fang et al. ${ }^{48}$ described a nanoliter-scale oil-airdroplet (OAD) chip for achieving multistep complex sample pretreatment and injection for single-cell proteomic analysis in the shotgun mode. As pioneers in the field of proteome analysis, Love et al..$^{49-51}$ have reported a series of research work on the detection of cytokine secreting from single cells. They also invented a microengraving method for generating microarrays that consist of small volume microwells in an array format. ${ }^{52}$ Through this method, individual cells were isolated, and the produced antigen-specific antibodies were selected rapidly by a microengraved substrate and sandwich-type immunoassay.

\subsection{Analysis of metabolite and metabolome}

Progress in genome, transcriptome and proteome has laid a foundation of cellular processes at the gene and protein levels for a systemic analysis. However, instruments are still needed for a reliable and sensitive analysis of intracellular small molecules, the so called-metabolome. Single-cell metabolome analysis as an emerging area answers basic biological questions and has important application in exploring directed evolution, drug toxicity, and cancer. ${ }^{53}$ Noninvasive analysis of metabolism at the single cell level, especially popular in evaluating cellular physiology for many applications. For example, Urbanski et al. ${ }^{54}$ developed an automated system for noninvasive metabolic profiling of single preimplantation embryos by microfluidic device (Fig. 4A). The structures of the metabolite detection chip were multichannel and the chip was fabricated by multilayer soft lithography. Due to the inherent flexibility of this system, the device was easily adapted for any $\mathrm{NAD}(\mathrm{P})$ H-based enzymatic assays. Moreover, this system could be applied as an independent device in which media samples were loaded or integrated into microfluidic culture systems for online, real-time metabolic evaluation. As the significance of metabolic activities profiling of embryos, Heo et al. ${ }^{55}$ also used a computerized microfluidic device with actuation based on deformation for real-time culture and automated periodic analyses of embryo metabolism. 

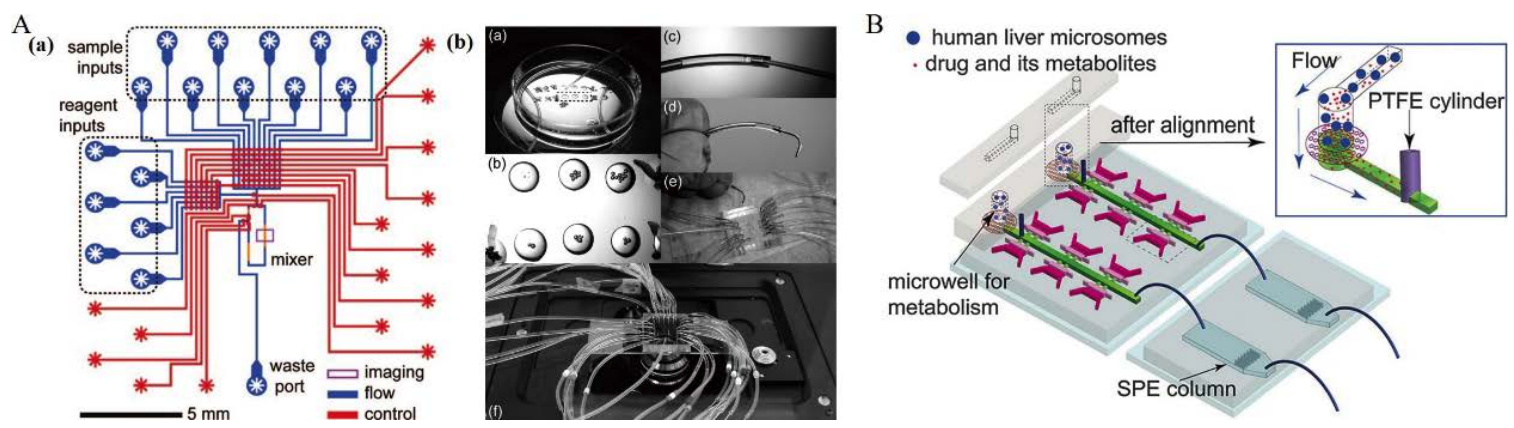

Fig. 4 Microfluidic device for single-cells metabolome profiling. (A) (a) Structural illustration of the microfluidic metabolite detector fabricated using multilayer soft lithography. (b) Sample and reagent loading sequence and the image of the metabolite detection. Adapted with permission from Ref. 54, Copyright 2008 American Chemical Society. (B) Schematic of an integrated microfluidic device for metabolism profiling. Adapted with permission from Ref. 60, Copyright 2014 The Royal Society of Chemistry.

As a powerful analytical technology, mass spectrometry (MS) has the capacity to label-free detect unknown molecules from inorganic elements to organic molecules with molecularly specific chemical information, especially suitable for identifying biomolecular structures, such as cell secretions and drug metabolites. In concerning chip-MS system, Lin's group has designed an on-chip intestine-liver model for multiple drug absorption and metabolism behavior simulation. ${ }^{56}$ In addition, Lin et al..$^{57-59}$ have reported some other work on single-cell analysis, and have written a number of reviews in this field. In the past few years, various new chemical entities (NCEs) and screenable drug targets emerged quickly. But only few of them were translated into new drug approvals successfully, in part because of negative biological consequences of drug metabolism and results in toxicity. Thus, early characterization of the metabolic fate and induced toxicity is an essential and significant part of drug metabolism research. Liu's group developed a novel multi-layer microfluidic device for the characterization of drug metabolism and their cytotoxicity assays in human liver microsomes (HLMs) during the same experiment (Fig. 4B). ${ }^{60}$ In this assay, metabolite generation, sample pretreatment, and online detection could be accomplished at the same integrated microfluidic system coupled with ESI-Q-TOF MS. Pestana et al. ${ }^{61}$ developed a biomimetic microfluidic device model together with liquid chromatography mass spectrometry (LCMS) for the metabolic profiling of human endothelial cells and its inhibition by the antimalarial drug chloroquine (CLQ).

\section{Single-cell ex vivo}

Since the Human Cell Atlas program was proposed, a new emerging challenge of cellomics, aiming to differentiate a variety of biochemical heterogeneity characteristics in a single cellular resolution systems, which will play an important role in discovering the differences among population cells and explaining their physiological behaviors in a specific environment. However, the most commonly used cellomics techniques are always based on a static or destructive principle, which encounters the difficulty of acquiring information at a continuous time point, high-throughput and precise manipulation in extracellular environment. Microfluidic devices have proven to be favorable tools for rapid and high-through manipulation, which is permitted for single-cell studies combined with traditional detection techniques. The most common are optical and electrochemical analytical methods.

\subsection{Optical analysis}

Cell signaling is a fast, dynamic, and complex process, which controls a variety of critical physiological functions. Optical techniques are popular in cell signaling with the capacity to directly visualize the morphology of cells and able to analyze target molecules with sub-micrometer resolution. However, individual optical analysis suffers from limited throughput at the single-cell level, and lack of precise fluid manipulation. The coupling of optical tweezers with microfluidic devices provides a precise control for live-cell positioning and dynamic stimulation in the microenvironment. Not long ago, our group presented a new strategy, ${ }^{62}$ termed dynamic microfluidic cytometry (DMC) for single-cell cellomics studies. It is known that normal flow cytometry can realize single-cell analysis with high throughput, but lack of dynamics study. The microplate system can be used to acquire dynamic information on a large number cells, and can not be used for single-cell resolution. From the traditional optical microscopy image, single-cell dynamics can be achieved, but the fluorescence of large number cells can not be quantified under a particular vision at one moment. The DMC system combined the strengths of normal flow cytometry, microfluidics and fluorescence microscopy, and realized the high-throughput probing of $G$ protein-coupled receptor (GPCR) signaling in single-cell resolution (Fig. 5A). Before that, we also reported a series studies on high-throughput single-cell cellomics. ${ }^{63-65}$ Besides cell signaling, the biochemical reactions ex vivo is also important for cellomics analysis. The most advanced and extensively utilized optical techniques for biochemical reactions and studies of cell biology is fluorescence resonance energy transfer (FRET). The FRET technique, which involves energy transfer from a donor fluorophore to an acceptor molecule, is usually combine with molecular beacon and aptamer for target molecular recognition. For example, Tang's group ${ }^{66}$ proposed an aptamer and nicking enzyme-assisted signal amplification to realize the membrane protein recognition of Hela cells on a microfluidic device (Fig. 5B). In this assay, they designed a molecular beacon (MB) labeled with a fluorophorequencher pair attached to its ends, and the MB contains three fragments: PTK7 specific aptamer, DNA linker and a segment for amplification reaction. The MB incubated with Hela cells, the aptamer could bind to the PTK7 on the cell membrane and a nicking enzyme assisting signal amplification was applied. Ultimately, a highly sensitive and homogeneous detection system for membrane protein on individual cell was achieved inside microfluidic droplets.

Recently, efforts have been directed toward the label-free 

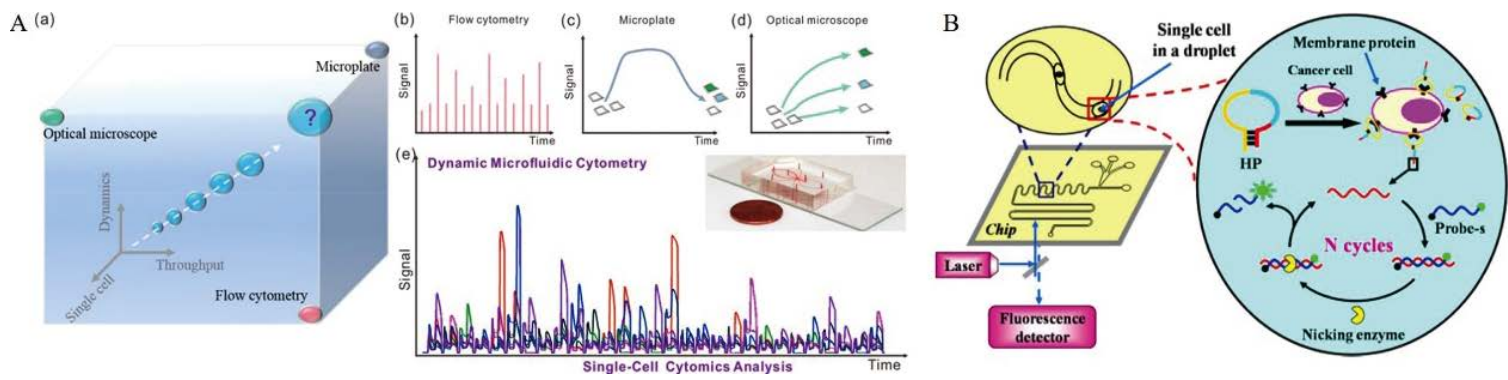

Fig. 5 Optical methods of single-cells analysis. (A) (a) Three-dimensional relation diagram of traditional methods for cell signaling analysis. (b-d) Typical signals obtained by flow cytometry, microplate, and optical microscope, respectively. (e) A typical trend of dynamic calcium signals of the Fluo-3/AM-loaded HeLa cells under ATP stimulation by dynamic microfluidic cytometry (DMC). Adapted with permission from Ref. 62, Copyright 2018 American Chemical Society. (B) Structural illustration of the microfluidic droplets assay and the process of enzyme-assisted signal amplification system in single-cells. Adapted with permission from Ref. 66, Copyright 2014 American Chemical Society.

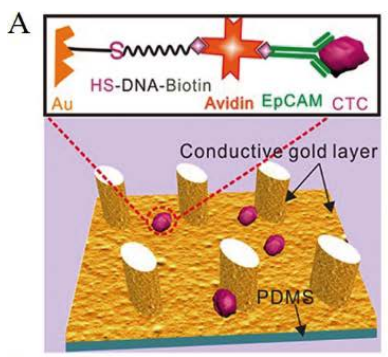

B (a)
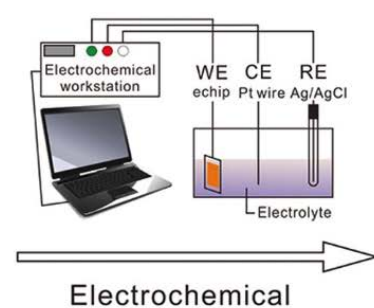

Electrochemical release/ lyse
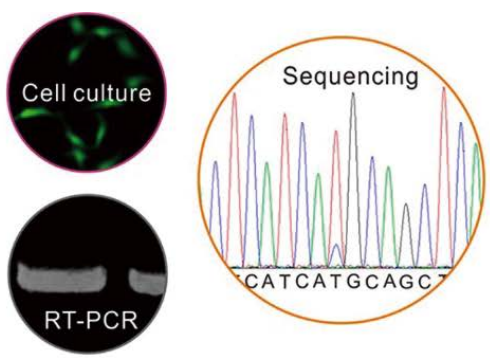

(b)
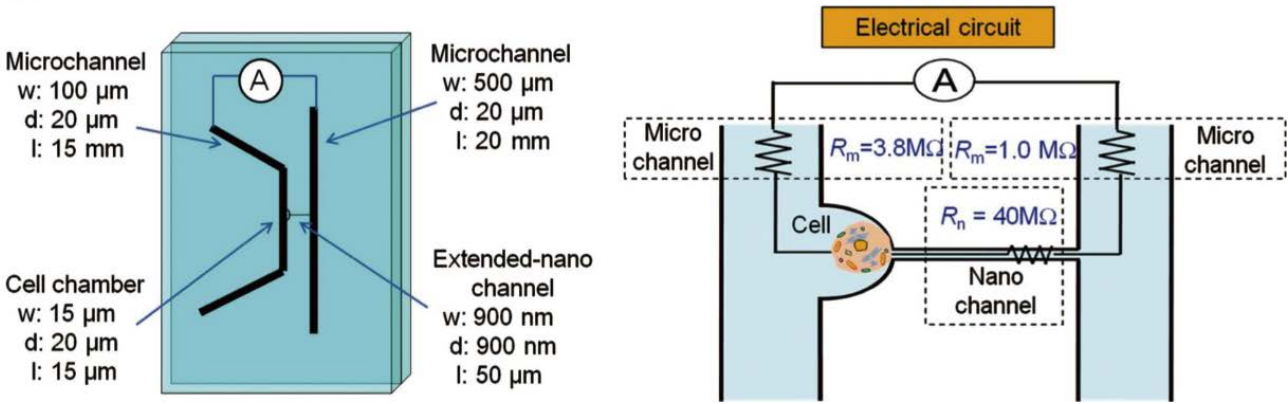

Fig. 6 Electrochemical methods of single-cells analysis. (A) The schematic of the microchip for the electrochemical capture, release, lysis and the analysis of the circulating tumor cells. Adapted with permission from Ref. 72, Copyright 2017 American Chemical Society. (B) Principle of electric resistance measurement for cell isolation and viability check: (a) chip design, (b) the equivalent circuit. Adapted with permission from Ref. 76, Copyright 2016 The Japan Society for Analytical Chemistry.

discrimination of cells. In light of this, Casabella et al. ${ }^{67}$ reported an automated microfluidic platform for the discrimination of live prostate epithelial cell based on laser tweezers Raman spectroscopy. Also a simple and inexpensive microfluidic flow chamber was introduced for allowing single cells to be optically trapped and analyzed. Stojanović et al. ${ }^{68,69}$ utilized surface plasmon resonance imaging for detecting and quantifying secreted antibodies from single hybridoma cells. For label-free and specific biomolecule detection at low lost, Agnarsson et al. ${ }^{70}$ invented a light-scattering microscopy technique integrated with a waveguide chip for imaging faint objects, such as sub-100 nm lipid vesicles and live cells.

\subsection{Electrochemical analysis}

Besides optical methods for cell analysis, electrochemicalbased microfluidic chips have also attracted much attention due to the relative paucity of electroactive contaminants and the sensitivity of microelectrodes to a wide range of biomolecules. For quantitative measurements of reactive oxygen and nitrogen species in living cells with excellent electrochemical and mechanical performances, Huang et al. ${ }^{4}$ produced cylindrical nanowire electrodes based on the design of a unique SiC-coreshell. Recently, our group demonstrated a rhipsalis (cactaceae)like hierarchical structure based three-dimensional microfluidic chip for highly efficient isolation of circulating tumor cells (CTCs). ${ }^{71}$ Next we utilized the electrochemical microchip (echip) to capture, release, lysis, and analysis of rare cancer 
cells with high efficiency (Fig. 6A). ${ }^{72}$ The multifunctional echip was made up of a glass slide and PDMS micropillar array which was plated a gold layer by electroless plating method. Due to the hierarchical structures of the electroconductive gold layer, the echip could be applied to release and lyse the captured CTCs for downstream biological studies, such as the amplification of individual transcripts by RT-PCR and futher genotyping analysis. For CTCs specific identification and capture, Safaei et al. $^{73}$ also reported a sensitive electrochemical method integrated with a microfluidic cell capture system and an electrochemical enzyme-linked immunosorbent assay (ELISA) to isolate and detect cancer cells on-chip. Except for CTC assay, electrochemical techniques are available for the research of neuronal cells by monitoring the release of neurotransmitters. For instance, Liu et al. ${ }^{74}$ reported an approach based on Au nano electrodes with high spatial resolution for the detection of the release of dopamine from rat pheochromocytoma cells.

Cellular impedance measurements have attracted wide attention since it can reveal the cytotoxic effects of exotic substances and cell response to drug treatment. The conventional methods of cell-impedance measurements generally study the behavior of cell populations on large-sized electrodes, and provide data on the collective response of cells to a given physiological condition. To measure the impedance of a single cell, the measuring electrodes must have a small size in order to execute accurate single-cell measurements. For this purpose, Guo et al. ${ }^{75}$ developed a novel electrode-based microarray chip used for measuring the adhering behavior of a single Hela cell by detecting its impedance spectra in situ. Besides, Lin et al. ${ }^{76}$ reported an extraordinary study that monitored the living cell viability by electric resistance measurement on a novel microfluidic platform. As shown in Fig. 6B, on the integrated single cell chamber and extended nano channel, a single cell could be isolated and cultured for more than $12 \mathrm{~h}$ by small pressure driven. Electric resistance measurements of cells have been carried out, indicating that extended-nano chips provided a technical platform for monitoring cell viability in real time.

\section{Single-cell in vivo}

Cells are the most basic entities for an organism's biological activity. In turn, understanding and studying the biological processes in multicellular organisms is important to analyze and characterize cellular physiological phenomena. Most recently, a microfluidic device has emerged as a powerful instrument for neurology and behavior investigations of small model organisms, such as C. elegans, zebrafish and Drosophila. In view of this, our group have reported a series of studies about the $C$. elegans on microfludic chips, ${ }^{77-80}$ one of these studies is shown in Fig. 7A. In this assay, we developed a novel semi-open PDMS microfluidic system for micro-manipulation of C. elegans and investigation of cell-cell communication in vivo. A piece of PDMS was abscised off the microfluidic chip to form an open chamber, and C. elegans were immobilized on the side wall of the chamber by suction. Through external microinjection of thapsigargin, individual intestinal cells of the immobilized C. elegans were stimulated. At the same time, the calcium dynamics in the intestinal cells was monitored by fluorescence imaging and the intercellular calcium wave (ICW) was observed in the intestinal cells. Furthermore, the mechanism of intercellular calcium signaling was investigated by analyzing the ICW propagation. Since C. elegans is a well-established model animal for studying the biological processes in organisms, Bosari et al. ${ }^{81}$ performed a microfluidic platform capable of acquiring high-throughput imaging of subcellular features in C. elegans with lifelong high-resolution. Zhu et al. ${ }^{82}$ presented a microfluidic impedance cytometry for measuring $C$. elegans and identifying their developmental stages.

Zebrafish with high genomic homology to humans is a simple vertebrate model organism that offers multiple applications in fundamental and biomedical research. ${ }^{74-76}$ The observation of zebrafish larvae's behavior in response to various stimuli is of interest in the field of nervous science. For instance, Peimani et al. ${ }^{83}$ developed a versatile microfluidic platform to study the rheotaxis and electrotaxis behavior of zebrafish larvae. As shown in Fig. 7B, they introduced the customized system for the electrical stimulation of zebrafish larvae inside a controllable environment. Through the quantitative analysis of larvae's electrotaxis movement towards the anode, they observed that the electrotaxis response diminished significantly at nighttime, while being enhanced significantly when exposing the larvae to dopamine agonists. Besides C. elegans and zebrafish, Drosophila is also a widely used organism with a simple neuronal system and short developmental period. Lu's group developed an optimized microfluidic device with dimensions of the embryo focusing and resistance channel for precise perturbation of live Drosophila embryos and monitoring the microenvironment of embryos responses to external stimuli, for example, gas-phase stimuli to the live embryos on the load device (Fig. 7C). ${ }^{84}$ In addition, they programmed software that could automatically segment confocal microscopy images of live embryo development for a simple, accurate morphometric analysis and data extraction. Ghaemi et al. ${ }^{85}$ have also made some effort to study the Drosophila behavior based on microfludic devices. For example, they showed a microfluidic micro-injector for toxicological and developmental studies in Drosophila embryos. In another work, they investigated a series of microfluidic clamps for intact immobilization and imaging of Drosophila larva's central nervous system. ${ }^{86}$

\section{Conclusions}

Studying single cells from different dimensions is a vital path to understand the complex organization of life. Single-cell analysis also helps to dissect the cellular biological processes and interactions of cellular physiological phenomena in multicellular organisms. An amazing variety of different methods and techniques already exist to study the morphology, composition and functions of single cells with respectable advantages such as high effective and sensitive. However, most of these approaches always encounter the difficulty of isolating, sorting and operating on a single cell in the process of sample preparation. The combination of traditional single-cell analysis methods with microfluidic system can solve these problem well. In this review, a microfluidic based analytical method for single-cell analysis at different dimensions: in vitro, ex vivo, in vivo were discussed. At last, for single cell analysis with microfluidic devices in near future, there are several outlooks: (1) develop a complete integration system with high-throughput and fullautomatic are required towards more precise single-cell, even subcellular analysis; (2) developing an intelligent microfluidic platform is required for vast single cell analysis with precise spatial localization in complex organism; (3) develop novel microfluidic systems for the simultaneous analysis of -omics (e.g. transcriptomics, proteomics and metabolomics) on an individual single cell. 
A (a)
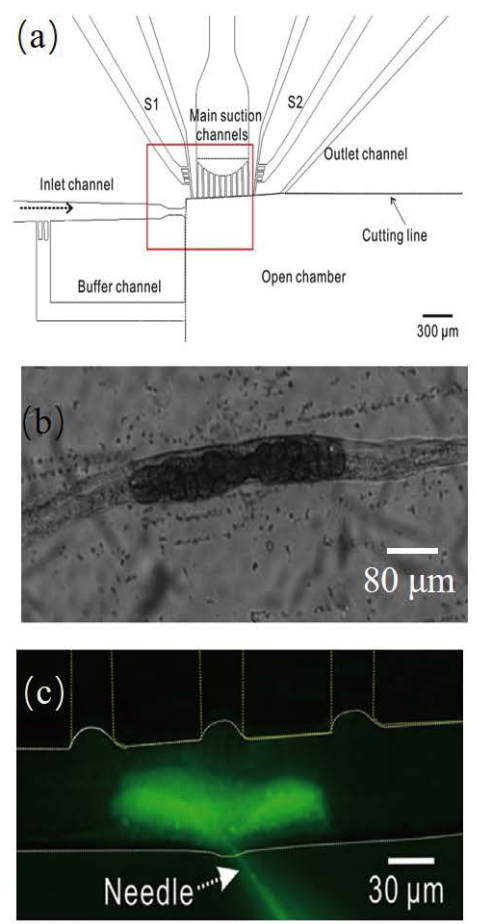

B
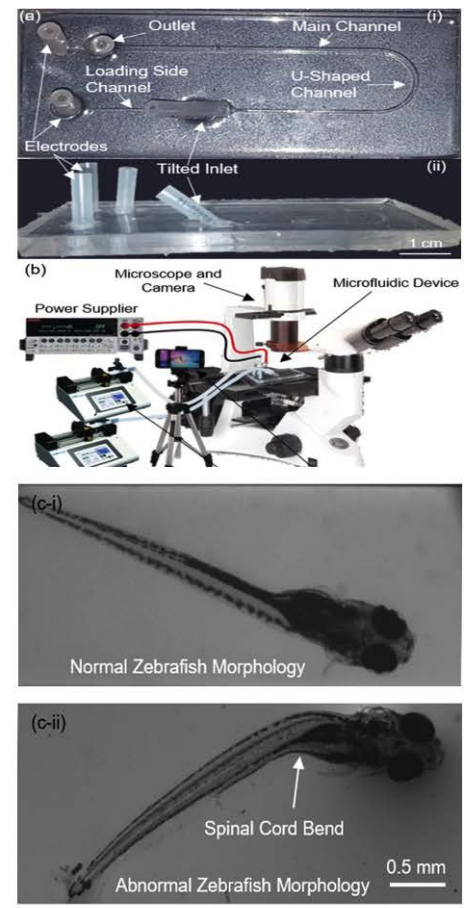

$\mathrm{C}$
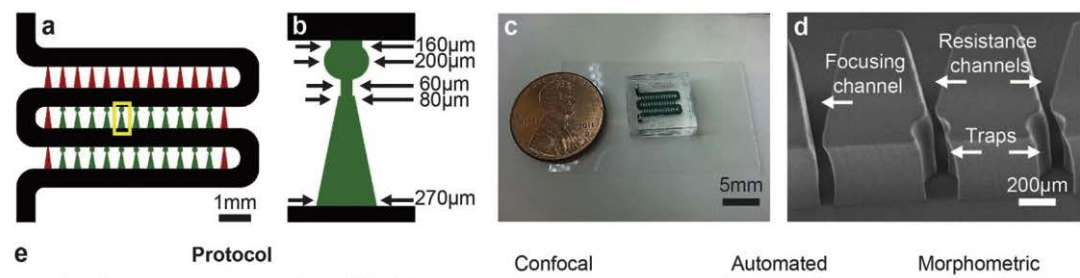

Confocal $\begin{array}{cc}\text { Confocal } & \text { Automated } \\ \text { Microscopy } & \text { Segmentation }\end{array}$ Segmentation Morphometric Analysis

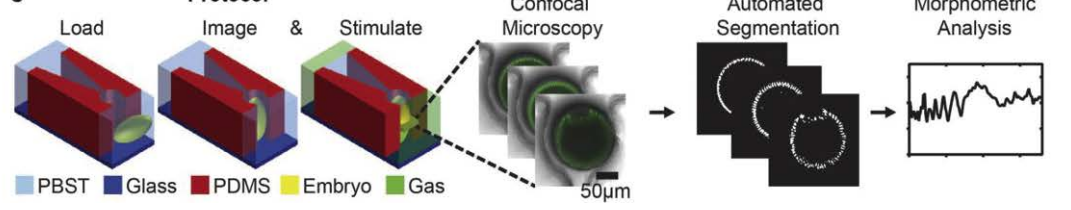

Fig. 7 (A) (a) The schematic of the semi-open microfluidic device, (b) Microscope image of the C. legans after immobilized on the agarose pad in the microfluidic chip for $10 \mathrm{~min}$, (c) The fluorescence image of the single intestinal cells in C. elegans microinjected with FITC. Adapted with permission from Ref. 77, Copyright 2013 Elsevier B.V. (B) (a) The top (i) and side (ii) views of the microfluidic chip, (b) The experimental setup of the electrotaxis study of zebrafish larvae, (c-i) Images of a normal zebrafish morphology and (c-ii) abnormal zebrafish morphology after exposure to $15 \mu \mathrm{A}$ electric current. Adapted with permission from Ref. 83, Copyright 2018 American Institute of Physics. (C) $(a-d)$ The schematic of the integrated platform in different ways. (e) The process of the morphometric analysis of live Drosophila embryos on the load device. Adapted with permission from Ref. 84, Copyright 2015 Springer Nature Publishing AG.

\section{Acknowledgements}

This work is supported by the National Natural Science Foundation of China (31870856, 31870854, 21775049 and 31700746), the National Key R\&D Program of China (2016YFF0100801 and 2017YFA0700403) and China Postdoctoral Science Foundation funded project (2018T110753 and 2018M630847).

\section{References}

1. L. M. Zahn, Science, 2017, 358, 57.

2. Y. Guo, Z. Li, W. Su, L. Wang, Y. Zhu, and J. Qin, Artif.
Organs., 2018, 42, 1196.

3. D. Cohen, J. A. Dickerson, C. D. Whitmore, E. H. Turner, M. M. Palcic, O. Hindsgaul, and N. J. Dovichi, Annu. Rev. Anal. Chem., 2008, 1, 165.

4. X. Zhang, Q. Qiu, H. Jiang, F. Zhang, Y. Liu, C. Amatore, and W. Huang, Angew. Chem., Int. Ed., 2017, 129, 13177.

5. Y. Song, T. Tian, Y. Shi, W. Liu, Y. Zou, T. Khajvand, S. Wang, Z. Zhu, and C. Yang, Chem. Sci., 2017, 8, 1736.

6. Y. Wang, H. Wang, P. Deng, W. Chen, Y. Guo, T. Tao, and J. Qin, Lab Chip, 2018, 18, 3606.

7. C. L. Brown, V. Fleischauer, and J. Heo, Anal. Sci., 2017, 33, 525 .

8. J. Nakanishi, T. Takarada, K. Yamaguchi, and M. Makea, Anal. Sci., 2008, 24, 67.

9. Y. Pei, X. Wang, W. Huang, P. Liu, and L. Zhang, Cellulose, 
2013, 20, 1897.

10. F. Fan, H. Shen, G. Zhang, X. Jiang, and X. Kang, Clin. Chim. Acta, 2014, 431, 113.

11. N. Ye, M. Wang, J. Qin, and B. Lin, Biomed. Microdevices, 2010, 12, 513.

12. L. T. H. Kao, L. Shankar, T. G. Kang, G. Zhang, G. K. I. Tay, S. R. M. Rafei, and C. W. H. Lee, Biosens. Bioelectron., 2011, 26, 2006.

13. A. Hibara, M. Fukuyama, M. Chung, C. Priest, and M. A. Proskurnin, Anal. Sci., 2016, 32, 11.

14. S. Lo and D. Yao, Int. J. Mol. Sci., 2015, 16, 16763.

15. X. Feng, B. F. Liu, J. Li, and X. Liu, Mass. Spectrom. Rev., 2015, 34, 535 .

16. Z. Wang, M. Gerstein, and M. Snyder, Nat. Rev. Genet., 2009, 10, 57.

17. L. He, A. Kniss, A. San-Miguel, T. Rouse, M. L. Kemp, and H. Lu, Lab Chip, 2015, 15, 1497.

18. T. H. Ong, D. J. Kissick, E. T. Jansson, T. J. Comi, E. V. Romanova, S. S. Rubakhin, and J. V. Sweedler, Anal. Chem., 2015, 87, 7036.

19. Y. Sakuta, I. Takehara, K. Tsunoda, and K. Sato, Anal. Sci., 2018, 34, 1073.

20. M. K. Alam, E. Koomson, H. Zou, C. Yi, C. Li, T. Xu, and M. Yang, Anal. Chim. Acta, 2018, 1044, 29.

21. H. Zhang, X. Cui, J. Bi, S. Dai, and H. Ye, Eng. Life Sci., 2015, 15, 582 .

22. A. Giulietti, L. Overbergh, D. Valckx, B. Decallonne, R. Bouillon, and C. Mathieu, Methods, 2001, 25, 386.

23. T. Kalisky, S. Oriel, T. H. B. Lev, N. B. Haim, A. Trink, Y. Wineberg, I. Kanter, S. Gilad, and S. Pyne, Brief. Funct. Genomics, 2018, 17, 64.

24. M. Hosokawa, Y. Nishikawa, M. Kogawa, and H. Takeyama, Sci. Rep-UK, 2017, 7, 5199.

25. M. M. Kiss, L. O. Donnelly, N. R. Beer, J. Warner, C. G. Bailey, B. W. Colston, J. M. Rothberg, D. R. Link, and J. H. Leamon, Anal. Chem., 2008, 80, 8975.

26. K. A. Heyries, C. Tropini, M. VanInsberghe, C. Doolin, O. I. Petriv, A. Singhal, K. Leung, C. B. Hughesman, and C. L. Hansen, Nat. Methods, 2011, 8, 649.

27. S. Karra and W. Gorski, Anal. Chem., 2013, 85, 10573.

28. T. X. Hu, X. H. Zhang, L. Zhang, W. Wen, and S. F. Wang, Biosens. Bioelectron., 2016, 77, 451.

29. N. Tsuyama, H. Mizuno, and T. Masujima, Anal. Sci., 2011, 27, 163.

30. K. Sato, Anal. Sci., 2015, 31, 867.

31. Z. Wang, M. Gerstein, and M. Snyder, Nat. Rev. Genet., 2009, 10, 57.

32. F. Tang, C. Barbacioru, Y. Wang, E. Nordman, C. Lee, N. Xu, X. Wang, J. Bodeau, B. B. Tuch, A. Siddiqui, K. Lao, and M. A. Surani, Nat. Methods, 2009, 6, 377.

33. F. Tang, C. Barbacioru, E. Nordman, B. Li, N. Xu, V. I. Bashkirov, K. Lao, and M. A. Surani, Nat. Protoc., 2010, 5 , 516.

34. A. M. Streets, X. Zhang, C. Cao, Y. Pang, X. Wu, L. Xiong, L. Yang, Y. Fu, L. Zhao, F. Tang, and Y. Huang, Proc. Natl. Acad. Sci. U. S. A., 2014, 111, 7048.

35. L. Huang, F. Ma, A. Chapman, S. Lu, and X. S. Xie, Annu. Rev. Genomics Hum. Genet., 2015, 16, 79.

36. H. C. Fan, G. Fu, and S. P. Fodor, Science, 2015, 347, 1258367.

37. S. M. Prakadan, A. K. Shalek, ans D. A. Weitz, Nat. Rev. Genet., 2017, 18, 345.

38. A. M. Klein, L. Mazutis, I. Akartuna, N. Tallapragada, A. Veres, V. Li, L. Peshkin, D. A. Weitz, and M. W. Kirschner, Cell, 2015, 161, 1187.
39. M. Wu and A. K. Singh, Curr. Opin. Biotech., 2012, 23, 83.

40. S. Hu, D. A. Michels, M. A. Fazal, C. Ratisoontorn, M. L. Cunningham, and N. J. Dovichi, Anal. Chem., 2004, 76, 4044.

41. J. M. Irish, R. Hovland, P. O. Krutzik, O. D. Perez, O. Bruserud, B. T. Gjertsen, and G. P. Nolan, Cell, 2004, 118, 217.

42. N. J. Dovichi and S. Hu, Curr. Opin. Chem. Biol., 2003, 7, 603.

43. J. R. Newman, S. Ghaemmaghami, J. Ihmels, D. K. Breslow, M. Noble, J. L. DeRisi, and J. S. Weissman, Nature, 2006, 441, 840.

44. B. Huang, H. Wu, D. Bhaya, A. Grossman, S. Granier, B. K. Kobilka, and R. N. Zare, Science, 2007, 315, 87

45. A. J. Hughes, D. P. Spelke, Z. Xu, C. C. Kang, D. V. Schaffer, and A. E. Herr, Nat. Methods, 2014, 11, 749.

46. F. Xu, H. Zhao, X. Feng, L. Chen, D. Chen, Y. Zhang, F. Nan, J. Liu, and B. Liu, Angew. Chem. Int. Ed., 2014, 53, 6730.

47. D. Chen, F. Fan, X. Zhao, F. Xu, P. Chen, J. Wang, L. Ban, Z. Liu, X. Feng, Y. Zhang, and B. Liu, Anal. Chem., 2016, 88, 2466.

48. Z. Li, M. Huang, X. Wang, Y. Zhu, J. Li, C. C. L. Wong, and Q. Fang, Anal. Chem., 2018, 90, 5430.

49. Q. Han, E. M. Bradshaw, B. Nilsson, D. A. Hafler, and J. C. Love, Lab Chip., 2010, 10, 1391.

50. J. Choi, K. R. Love, Y. Gong, T. M. Gierahn, and J. C. Love, Anal. Chem., 2011, 83, 6890.

51. Q. Han, N. Bagheri, E. M. Bradshaw, D. A. Hafler, D. A. Lauffenburger, and J. C. Love, Proc. Natl. Acad. Sci. U. S. A., 2012, 109, 1607.

52. Q. Han, E. M. Bradshaw, B. Nilsson, D. A. Hafler, and J. C. Love, Lab Chip., 2010, 10, 1391.

53. G. B. Salieb-Beugelaar, G. Simone, A. Arora, A. Philippi, and A. Manz, Anal. Chem., 2010, 82, 4848.

54. J. P. Urbanski, M. T. Johnson, D. D. Craig, D. L. Potter, D. K. Gardner, and T. Thorsen, Anal. Chem., 2008, 80, 6500.

55. Y. S. Heo, L. M. Cabrera, C. L. Bormann, G. D. Smith, and S. Takayama, Lab Chip, 2012, 12, 2240.

56. M. Jie, H. Lin, Z. He, H. Liu, H. Li, and J. Lin, Sci. China Chem., 2018, 61, 236.

57. Q. Huang, S. Mao, M. Khan, and J. Lin, Analyst, 2019, 144, 808

58. M. Khan, S. Mao, W. Li, and J. Lin, Chem.-Eur. J., 2018, 24, 15398.

59. F. Chen, L. Lin, J. Zhang, Z. He, K. Uchiyama, and J. Lin, Anal. Chem., 2016, 88, 4354

60. Q. Wu, D. Gao, J. Wei, F. Jin, W. Xie, Y. Jiang, and H. Liu, Chem. Commun., 2014, 50, 2762.

61. C. R. Pestana, A. C. Urbaczek, J. V. Alberici, G. J. Rodrigues, and E. Carrilho, Life. Sci., 2017, 172, 42.

62. P. Chen, S. Yan, J. Wang, Y. Guo, Y. Dong, X. Feng, X. Zeng, Y. Li, W. Du, and B. Liu, Anal. Chem., 2019, 91, 1619.

63. Y. Li, D. Chen, Y. Zhang, C. Liu, P. Chen, Y. Wang, X. Feng, W. Du, and B. Liu, Sens. Actuators, B, 2016, 225, 563.

64. P. Chen, Y. Guo, X. Feng, S. Yan, J. Wang, Y. Li, W. Du, and B. Liu, Anal. Chem., 2017, 89, 9209.

65. P. Chen, X. Feng, S. Yan, Y. Guo, J. Wang, Y. Li, D. Chen, W. Du, and B. Liu, Sens. Actuators, B, 2018, 263, 281.

66. L. Li, Q. Wang, J. Feng, L. Tong, and B. Tang, Anal. Chem., 2014, 86, 5101.

67. S. Casabella, P. Scully, N. Goddard, and P. Gardner, Analyst, 2016, 141, 689. 
68. B. Agnarsson, A. Lundgren, A. Gunnarsson, M. Rabe, A. Kunze, M. Mapar, L. Simonsson, M. Bally, V. P. Zhdanov, and F. Höök, ACS Nano, 2015, 9, 11849.

69. B. Agnarsson, H. K. Wayment-Steele, F. Höök, and A. Kunze, Nanoscale, 2016, 8, 19219.

70. I. Stojanović, T. J. G. vander Velden, H. W. Mulder, R. B. M. Schasfoort, and L. W. M. M. Terstappen, Anal. Biochem., 2015, 485, 112.

71. S. Yan, X. Zhang, X. Dai, X. Feng, W. Du, and B. Liu, Sens. Actuators, B, 2016, 8, 33457.

72. S. Yan, P. Chen, X. Zeng, X. Zhang, Y. Li, Y. Xia, J. Wang, X. Dai, X. Feng, W. Du, and B. Liu, Anal. Chem., 2017, 89, 12039.

73. T. S. Safaei, R. M. Mohamadi, E. H. Sargent, and S. O. Kelley, ACS Appl. Mater Inter, 2015, 7, 14165.

74. Y. Liu, M. Li, F. Zhang, A. Zhu, and G. Shi, Anal. Chem., 2015, 87, 5531.

75. X. L. Guo, R. Zhu, and X. L. Zong, Analyst, 2015, 140, 6571.

76. L. Lin, K. Mawatari, K. Morikawa, and T. Kitamori, Anal. Sci., 2016, 32, 75.
77. X. Zhao, F. Xu, L. Tang, W. Du, X. Feng, and B. Liu, Biosens. Bioelectron., 2013, 50, 28.

78. L. Hu, A. Ge, X. Wang, S. Wang, X. Yue, J. Wang, X. Feng, W. Du, and B. Liu, Biosens. Bioelectron., 2018, 110, 233.

79. L. Hu, J. Ye, H. Tan, A. Ge, L. Tang, X. Feng, W. Du, and B. Liu, Anal. Chim. Acta, 2015, 887, 155.

80. X. Wang, L. Tang, Y. Xia, L. Hu, X. Feng, W. Du, and B. Liu, Integr. Biol., 2013, 5, 728.

81. S. S. Bosari, J. Huayta, and A. S. Miguel, Lab Chip, 2018, $18,3090$.

82. Z. Zhu, W. Chen, B. Tian, Y. Luo, J. Lan, D. Wu, D. Chen, Z. Wang, and D. Pan, Sens. Actuators, B, 2018, 275, 470.

83. A. R. Peimani, G. Zoidl, and P. Rezai, Biomicrofluidics, 2018, 12, 14113.

84. T. J. Levario, C. Zhao, T. Rouse, S. Y. Shvartsman, and H. Lu, Sci. Rep-UK, 2016, 6, 21366.

85. R. Ghaemi, P. Arefi, A. Stosic, M. Acker, Q. Raza, J. J. Roger, and P. R. Selvaganapathy, Lab Chip, 2017, 17, 3898.

86. R. Ghaemi, P. Rezai, F. R. Nejad, and P. R. Selvaganapathy, Biomicrofluidics, 2017, 11, 34113. 